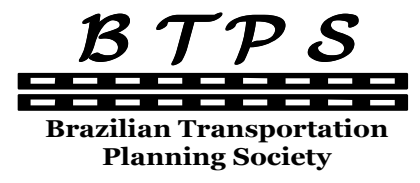

Journal of Transport Literature

Vol. 7, n. 4, pp. 225-246, Oct. 2013

Research Directory
JTL | RELIT

www.transport-literature.org

\title{
Modelagem de fatores da expansão urbana e padrões de viagens aplicando equações estruturais
}

[Urban sprawl and travel patterns modeling with structural equations]

\author{
Ronny Marcelo Aliaga Medrano*, Pastor Willy Gonzales Taco \\ Universidade de Brasília - Brasil
}

Submitted 5 Oct 2012; received in revised form 20 Jan 2013; accepted 21 Jan 2013

\begin{abstract}
Resumo
O objetivo do trabalho é analisar a relação dos efeitos espaciais entre expansão urbana e padrões de viagens baseadas em atividades, mediante a utilização da modelagem com equações estruturais. Foi elaborado um modelo causal que relaciona os fatores da expansão urbana de forma indireta com os padrões de viagens baseados em atividades através da formação de construtos latentes. Assim, a estrutura teórica do modelo "ABTUS (Activity-Based Travels of Urban Sprawl)" foi fundamentada em dois construtos latentes: i) produção de espaço; e, ii) características socioeconômicas. 0 modelo foi testado em caso de estudo de Brasilia-Distrito Federal para o ano 2000. A partir dos resultados obtidos foram reunidos indícios da relação entre expansão urbana e padrões de viagens baseadas em atividades. Cinco vetores de expansão urbana foram identificados e os padrões de viagens mais representativos foram baseados nas sequências de atividades Residência Trabalho e Residência Estudo. Portanto, para o caso de Brasília a centralização dos empregos e o restito espaço de moradia no centro da cidade, produzem maiores viagens nas expansões urbanas por trabalho e estudo.
\end{abstract}

Palavras-Chave: expansão urbana, viagens baseadas em atividades, modelagem com equações estruturais.

\begin{abstract}
The objective of this paper is to analyze the relationship between urban sprawl spatial effects and activity-based travel patterns through the use of structural equation modeling. It was developed a causal model that related urban sprawl factors indirectly with the activity-based travel patterns through the formation of latent constructs. The theoretical structure of the model "ABTUS (Activity-Based Travels of Urban Sprawl)" was based on two latent constructs: i) production of space, and ii) socioeconomic characteristics. The model was tested in a case study of Brasilia, Distrito Federal for 2000. From the results obtained were gathered evidence of the relationship between urban sprawl and activity-based travel patterns. Five vectors of urban expansion were identified and the most representative patterns of travel were based on the sequences of activities Housing-Work and Housing-Study. Therefore, in the case of Brasilia, the centralization of jobs and limited living space in the city center, are producing urban expansions with many travels for work and study.
\end{abstract}

Key words: urban sprawl, activity-based travel, structural equation modeling.

*Email: ronnymarcelonmt@gmail.com.

\section{Recommended Citation}

Medrano, R. M. A. and Taco, P. W. (2013) Modelagem de fatores da expansão urbana e padrões de viagens aplicando equações estruturais. Journal of Transport Literature, vol. 7, n. 4, pp. 225-246.

- JTL/RELIT is a fully electronic, peer-reviewed, open access, international journal focused on emerging transport markets and published by BPTS - Brazilian Transport Planning Society. Website www.transport-literature.org. ISSN 2238-1031. 


\section{Introdução}

Este artigo tem como objetivo analisar a relação entre fatores da expansão urbana e padrões de viagens baseados em atividades. O modelo "ABTUS (Activity-Based Travels of Urban Sprawl)" foi elaborado para testar estas relações mediante a técnica estatística Equações Estruturais ou "Structural Equation Modelling" (SEM). SEM é uma técnica que pode lidar com um grande número de variáveis endógenas e exógenas, e variáveis latentes (não observadas) especificadas como combinações lineares interdependentes. Assim, a aplicação do SEM permitiu a análise da relação entre fatores da expansão urbana e padrões de viagens através de processos latentes que foram combinados linearmente com suas respectivas variáveis indicadoras.

O transporte é essencial para o bem-estar social e econômico da população, tem estrita relação com o desenvolvimento econômico das cidades, principalmente nos países em desenvolvimento (Zhao, 2010). O rápido processo de expansão urbana gera mudanças nos transportes, gerando novos padrões de viagens, tais como o aumento das viagens motorizadas e das distancias de viagens (Gakenheimer, 1999; Kenworthy, 1995).

Observa-se que as realidades americanas, asiáticas e europeias, apresentam padrões de expansão distintos da realidade latino-americana. A falta de políticas, planos urbanos e controles efetivos da maioria das cidades produz expansões urbanas caóticas, impactando na qualidade de vida dos cidadãos. Poucas cidades brasileiras conseguiram estabelecer a expansão urbana controlada e integrada a um sistema de transporte eficiente, resultando em padrões de viagens representativos da configuração urbana, como é o caso da cidade de Curitiba/PR.

Conforme afirmam Giuliano e Narayan (2003), as relações entre expansão urbana e os padrões de mobilidade urbana variam em função do contexto e das condições das cidades. Como por exemplo, quando são consideradas as relações entre a densidade habitacional, dependência do veiculo privado e uso de transporte público urbano.

Definir a relação entre a expansão urbana e o transporte urbano é um tanto difícil, pois não existe um entendimento único sobre o tema. A expansão urbana não se resume apenas a um 
processo quantitativo de extensão territorial e dispersão da densidade populacional, também inclui mudanças qualitativas no ambiente urbano (Castells, 1974). Dentro deste contexto de organização do espaço na estrutura urbana, pode-se dizer que o transporte é um elemento importante que interage tanto com fatores quantitativos como qualitativos do sistema urbano, influenciando e sendo influenciado pelo processo de expansão urbana (Cervero, 1998).

Para Cervero (1998) e Ewing (1997) a expansão urbana tem uma influência importante no desenvolvimento do transporte; principalmente no que se refere à geração da demanda, dos tempos de viagem e da escolha modal. Além disso, políticas e investimentos em transportes, como melhorias na infraestrutura e na provisão de serviços de transporte público urbano, podem orientar o desenvolvimento da expansão urbana.

Há relevância na análise da relação entre fatores da expansão urbana e padrões de viagens baseados em atividades, em especial, porque a expansão urbana é um fenômeno que afeta todo tipo de cidade. Criando impactos negativos no sistema de transporte quando não é feito um planejamento integrado. Portanto, procura-se também fornecer uma ferramenta para o planejamento de transportes urbanos.

Os resultados encontrados permitem entender a importância da relação estrutural da covariância entre as características espaciais urbanas e as características dos indivíduos na geração de padrões de viagens baseados em atividades. Assim, Brasilia apresenta essa relação estrutural fortemente marcada com a segregação e expansão das urbanizações, a causa das estratégias, políticas e ações realizadas durante o crescimento desta cidade.

O presente trabalho está assim dividido: na Seção 1, aborda-se o referencial teórico sobre a modelagem da relação entre expansão urbana e padrões de viagens baseados em atividades. Na Seção 2, apresenta-se uma introdução à técnica estatística SEM e a formulação do modelo ABTUS. A seção 3 define as variáveis a ser utilizadas e sistematiza o modelo ABTUS. Na seção 4, apresenta-se a analise dos resultados e os indicadores de avaliação de ajusto do modelo. E por fim, nas Conclusões, estrutura-se uma avaliação da proposta de análise deste trabalho e da aplicação do modelo ABTUS. Também se avalia a importância da análise da relação entre fatores da expansão urbana e padrões para o planejamento de transportes urbanos. 


\section{Transporte e expansão urbana modelagem}

As formas de expansão urbana (dispersão suburbana e a constituição de metrópoles policêntricas) tendem a aumentar as distâncias de viagens e, principalmente, favorecem a maior dependência do uso de veículo privado e maiores custos de operação de transporte público. Além disso, a prestação de serviço de transporte público urbano ineficiente, considerando que: a demanda de transporte público urbano é baixa; a dispersão da demanda sobre o território é alta; e, o incremento da dispersão dos destinos é causado pela suburbanização das áreas de emprego (Cervero, 1989; Cervero e Kockelman, 1997; Newman e Kenworthy, 1999).

Os primeiros fatores a serem relacionados aos padrões de viagens são a densidade e sua distribuição na área urbana. Densidades baixas e dispersas estimulam maior dependência do veículo privado e geram maiores distâncias de viagem. Densidades altas e concentradas estimulam viagens curtas e maior uso de modos não motorizados e de transporte público (Friedman et al., 1994, Cervero, 1998; Duany et al., 2000; Steiner, 1994). Para Cervero e Kockelman (1997) e Ewing (1997) os maiores impactos nos padrões de viagens, além dos fatores socioeconômicos, ocorrem em função de três fatores da estrutura urbana, sendo eles: a densidade (ou a concentração, crescimento e distribuição populacional ou residencial); a diversidade (que é o uso de solo misto e ocupação urbana); e, o desenho urbano (ou o uso de solo misto e ocupação urbana).

Fatores da expansão urbana relacionados diretamente com a densidade (especificamente baixas densidades) e com a diversidade do uso de solo (residenciais versus industriais) influenciam de uma forma causal na formação dos padrões de viagens (Frank e Pivo, 1994; Camagni et al.2002; Ewing et al. 2002; e Travisi et al. 2010).

Nesse contexto, algumas modelagens empíricas foram desenvolvidas com o intuito de prover subsidio ao entendimento da relação entre a expansão urbana e os padrões de viagens observados. A maioria das pesquisas corresponde a países europeus, asiáticos e americanos. Um estudo comparativo entre Estados Unidos e Inglaterra foi elaborado por Giuliano e Narayan (2002). O objetivo era comparar como os fatores de forma urbana das expansões urbanas influenciavam nos padrões de viagens, além dos fatores socioeconômicos. Modelos de regressão linear foram construídos para comparar os fenômenos. 
Travisi et al. (2010) analisaram empiricamente a relação entre expansão urbana e os viagens diárias ao trabalho para sete áreas urbanas de Itália. A modelagem de relações entre o desenvolvimento espacial e fatores explicativos relacionados a mudanças na densidade urbana foram analisadas através da técnica multivariada de regressão de seções cruzadas.

Schwanen et al. (2001) analisaram os impactos das estruturas urbanas monocentricas e policentricas na escolha modal e as distâncias de viagem para diferentes atividades na Holanda. Modelos de regressão logística e modelos de regressão multivariada foram utilizados para estabelecer as relações entre as variáveis. Para um estudo específico GarcíaPalomares (2010) analisou a relação entre a expansão urbana e as viagens ao trabalho para a área metropolitana de Madrid-Espanha. Modelos de regressão lineal múltipla foram construídos para análise das relações entre os fatores de expansão urbana e de mobilidade.

Com base na revisão da literatura, e possível afirmar que existe uma influência de fatores da expansão urbana e socioeconômicos na formação dos padrões de viagens baseadas em atividades dos indivíduos. Tais fatores, segundo os estudos revisados, guardam uma relação linear com o comportamento de viagens (escolha do modo, distância percorrida, atividade principal). Assim, a aplicação de modelos que permitem entender o processo da expansão urbana e testar as relações dos fatores com os padrões de viagens são necessários à condução do presente trabalho.

Por ter aplicação ampla nas ciências do comportamento, a utilização da SEM como ferramenta de modelagem já se mostra muito promissora no que diz respeito à captura de intenções comportamentais, comportamento atual e especificação de testes de alternativas de relações de causalidade em comportamento para viagens (Golob, 2008). 


\section{Modelagem dos fatores de expansão urbana e padrões de viagens com equações estruturais (structural equation modelling)}

Nesta seção será dada uma introdução breve à técnica estatística utilizada neste trabalho. Equações estruturais ou "Structural Equation Modelling” (SEM) é uma técnica que pode lidar com um grande número de variáveis endógenas e exógenas, e variáveis latentes (não observadas) especificadas como combinações lineares das variáveis observadas. As variáveis latentes exógenas são mensuradas por indicadores cujos valores foram coletados. As variáveis latentes endógenas são também mensuradas por indicadores, mas também apresentam relações de regressão com as variáveis latentes exógenas. O método é confirmatório, e não exploratório, porque o modelador deverá formular o modelo em termos de um sistema de efeitos em uma única direção de uma variável sobre a outra (Golob, 2008), conforme representado na Equação 1:

$$
\boldsymbol{\eta}=\boldsymbol{\alpha}+\boldsymbol{B} \cdot \boldsymbol{\eta}^{\prime}+\Gamma \cdot \xi+\zeta
$$

Onde:

$\eta=$ Vetor que representa as variáveis latentes endógenas é de ordem mx 1 ;

$\eta^{\prime}=$ Vetor que representa as variáveis latentes endógenas mensuradas por indicadores e é de ordem mx1;

$B=$ Matriz de coeficientes de $\mathrm{m} \times \mathrm{n}$ que relaciona os $\mathrm{n}$ fatores exógenos com os $\mathrm{m}$ fatores endógenos;

$\Gamma$ = Matriz de coeficientes de $\mathrm{m} x \mathrm{~m}$ que relaciona os $\mathrm{m}$ fatores endógenos um com outro;

$\xi=$ Vetor de ordem nx1 e representa as n variáveis exógenas latentes;

$\zeta=$ Vetor de resíduos de $m x 1$ representa os erros na equação que relaciona $\eta$ e $B$;

$\alpha=$ Vetor de interceptos.

$$
\begin{aligned}
& x=\tau_{x}+\Lambda_{\mathrm{x}} \cdot \xi+\delta \\
& y=\tau_{y}+\Lambda_{\mathrm{y}} \cdot \xi+\varepsilon
\end{aligned}
$$

O objetivo do modelo SEM é a estimação dos coeficientes das Equações 1, 2 e 3, nos quais: 
$\tau_{x}$ e $\tau_{y}=$ representam os vetores dos interceptos;

$\delta$ e $\varepsilon=$ os vetores de erros não observáveis e não mensuráveis;

$\xi=$ o vetor de resíduos do modelo estrutural;

$\Lambda_{\mathrm{x}}$ e $\Lambda_{\mathrm{y}}=$ as matrizes de coeficientes de impactos das variáveis $\xi$ em X e $\eta$ em Y; e,

$\Gamma$ e $\mathrm{B}=$ as matrizes dos coeficientes de efeitos diretos de $\xi$ em $\eta$ e das interelações entre os construtos endógenos $(\eta)$.

\subsection{Concepção e sistematização do modelo Activity-Based Travels of Urban Sprawl (ABTUS)}

A formulação do modelo parte do pressuposto que os fatores espaciais da expansão urbana (FEXU) estão diretamente relacionados à geração de viagens por atividade (GVA). E as características socioeconômicas (CSE) dos indivíduos também estão diretamente relacionadas à geração de viagens.

O modelo se define da seguinte forma:

$$
F E X U \rightarrow G V A ; C S E \rightarrow G V A
$$

Considerando as relações apresentadas, pode-se definir que a geração de viagens por atividades é função dos fatores espaciais urbanos da expansão urbana e características socioeconômicas.

$$
G V A=f\{F E X U, C S E\}
$$

Assim, estes dois construtos latentes exógenos do comportamento da demanda de viagens são essenciais para explicar os padrões de viagens baseadas em atividades. As abordagens aplicadas só representam esta relação de uma forma linear entre as variáveis explicativas. $\mathrm{O}$ que indicaria que estas variáveis atuam de uma maneira independente entre si e seguem uma tendência linear para explicar a demanda de viagens. Mas na realidade, as variáveis não atuam de forma direta no comportamento da demanda de viagem, são na realidade explicativas de processos que influenciam a geração dos padrões de viagens baseadas em atividades.

Embora os processos sejam distintos e separados entre si, mas guardam relações de interdependência, interagindo simultaneamente e resultando no comportamento da demanda de viagens. A Figura 1 ilustra o processo de interação dos construtos latentes exógenos e 
endógeno, bem como as suas relações de interdependência e simultaneidade na geração de padrões de viagens.

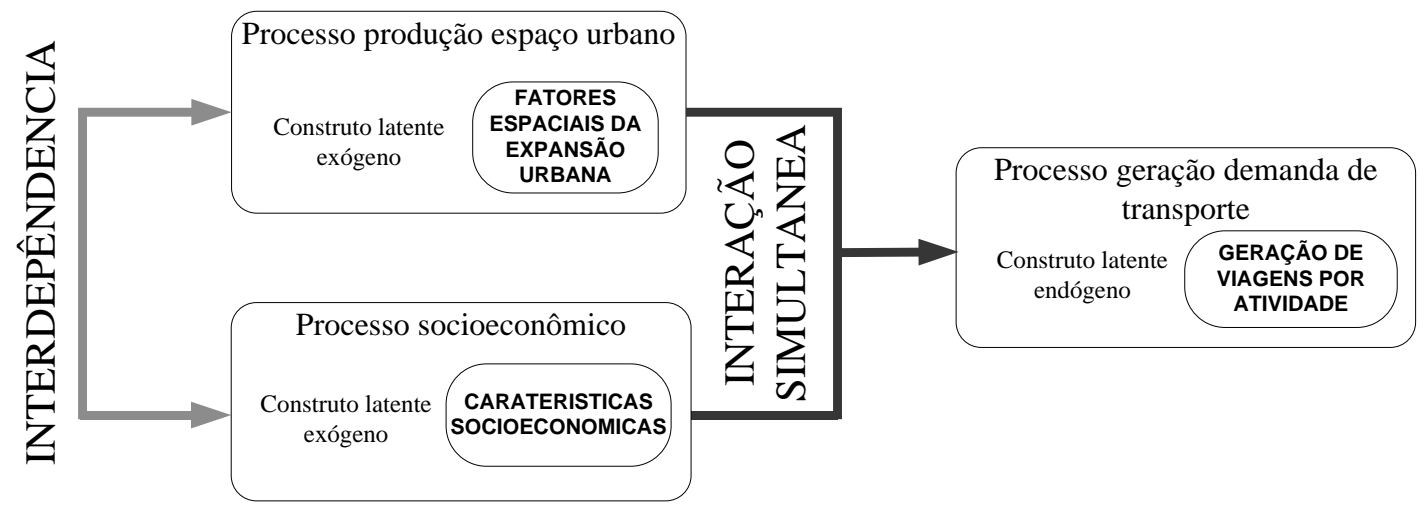

Figura 1 - Representação da interação entre processos ${ }^{11}$

A Equação 6 apresenta o modelo estrutural da seguinte forma:

$$
\boldsymbol{\eta}=\boldsymbol{\Gamma} \xi+\mathbf{B} \boldsymbol{\eta}^{\prime}+\zeta
$$

Onde:

$\eta=\{G V A\}=$ construto latente endógeno: Geração de viagens por atividades,

$\xi=\{F E X U, C S E\}=$ constructos latentes exógenos: Fatores espaciais da expansão urbana,

Características Socioeconômicas,

$\Gamma=\left\{\gamma_{11}, \ldots ., \gamma_{n m}\right\}=$ matriz de parâmetros associados com os constructos exógenos,

$\mathrm{B}=\left\{\beta_{11}, \ldots \ldots, \beta_{m n}\right\}=$ matriz de parâmetros associados com o constructo endógeno,

$\zeta=\left\{\zeta_{1}, \ldots . ., \zeta_{n}\right\}=$ vetor de termos de erros associados à estimação.

Assim, generalizando o modelo ABTUS como apresenta a Equação 7:

$$
G V A=\gamma_{11} F E X U+\gamma_{12} C S E+\zeta
$$

\footnotetext{
${ }^{1}$ Esta e as demais figuras são de desenvolvimento próprio dos autores.
} 


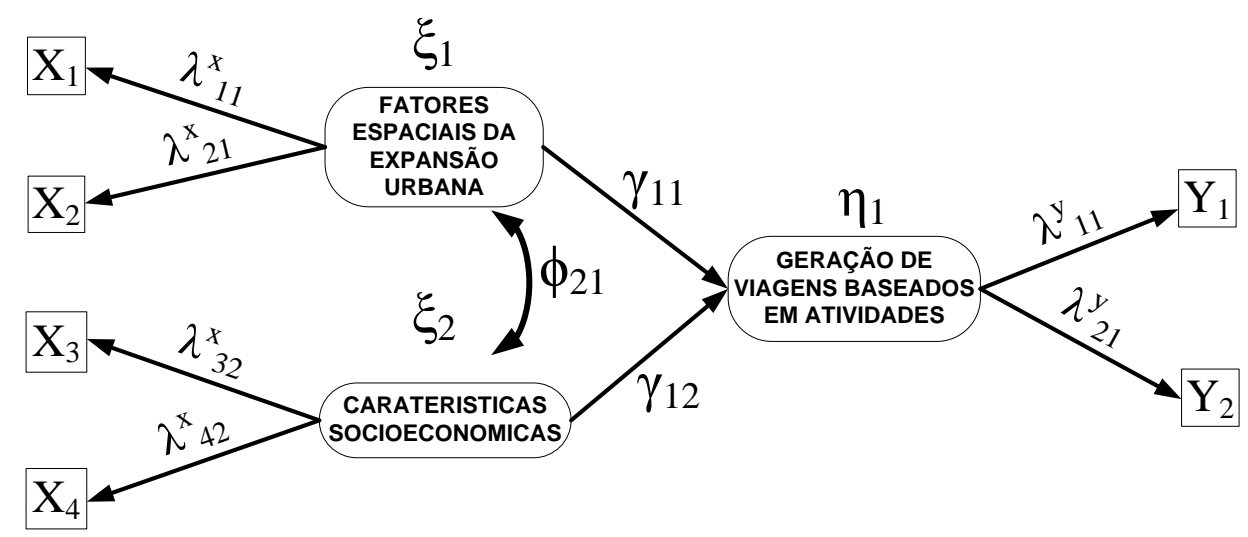

Figura 2 - Modelo Activity-Based Travels of Urban Sprawl (ABTUS)

A estrutura completa do modelo ABTUS é apresentada na Figura 2. Os construtos latentes e variáveis indicadoras, bem como os parâmetros a serem estimados, são listados nas Tabelas 1 e 2. A formulação dos construtos latentes e os indicadores não são apresentados em detalhe neste trabalho, mas são detalhados em Medrano (2012).

Tabela 1 - Descrição das variáveis latentes e parâmetros estruturais do ABTUS ${ }^{2}$

\begin{tabular}{cl}
\hline Variavel Latente & Descrição \\
\hline$\xi 1$ & Fatores espaciais urbanos da expansão urbana \\
$\xi 2$ & Características socioeconômicas \\
$\eta 1$ & Geração de viagens por atividade \\
\hline Parâmetro estrutural & \\
\hline \multirow{2}{*}{$\gamma 11$} & Impacto dos fatores espaciais da expansão urbana sobre o \\
& comportamento da demanda de viagem \\
$\gamma 12$ & Impacto das caraterísticas socioeconômicas sobre o comportamento da \\
& demanda de viagem \\
Covariância entre os fatores espaciais da expansão urbana e as \\
caraterísticas socioeconômicas
\end{tabular}

\footnotetext{
${ }^{2}$ Esta e as demais tabelas são de desenvolvimento próprio dos autores.
} 
Tabela 2 - Descrição das variáveis indicadoras e parâmetros estruturais do ABTUS

\begin{tabular}{|c|c|c|c|}
\hline \multicolumn{4}{|c|}{ Fatores espaciais da expansão urbana } \\
\hline Variavel indicadora & Notação & Parâmetro & Tipo de variável \\
\hline \multicolumn{3}{|l|}{ Densidade uso de solo } & \multirow{2}{*}{ Contínua } \\
\hline misto & $\mathrm{X} 1$ & $\lambda 11$ & \\
\hline Densidade residencial & $\mathrm{X} 2$ & $\lambda 21$ & Contínua \\
\hline \multicolumn{4}{|c|}{ Características socioeconômicas } \\
\hline Variavel indicadora & Notação & Parâmetro & Tipo de variável \\
\hline $\begin{array}{l}\text { Número de famílias } \\
\text { com renda acima de } \\
20 \text { salarios mínimos }\end{array}$ & $\mathrm{X} 5$ & $\lambda 53$ & Contínua \\
\hline $\begin{array}{c}\text { Número de famílias } \\
\text { com dois carros o } \\
\text { mais }\end{array}$ & $\mathrm{X} 6$ & $\lambda 63$ & Contínua \\
\hline \multicolumn{4}{|c|}{ Geração de viagens por atividade } \\
\hline Variavel indicadora & Notação & Parâmetro & Tipo de variável \\
\hline $\begin{array}{l}\text { Padrão de viagens } \\
\text { Residência Trabalho }\end{array}$ & $\mathrm{Y} 1$ & $\lambda 11$ & Contínua \\
\hline $\begin{array}{l}\text { Padrão de viagens } \\
\text { Residência Estudo }\end{array}$ & $\mathrm{Y} 2$ & $\lambda 21$ & Contínua \\
\hline
\end{tabular}

\section{3. $O$ contexto urbano do Distrito Federal}

Brasília apresenta uma estrutura polinucleada, tendo o Plano Piloto como centro e a localização das cidades satélites fora da bacia do lago do Paranoá, ideia que predominou durante os anos 60 e norteou as políticas restritivas de assentamento populacional no território do DF. No início dos anos 90, intensificaram-se os conflitos na ocupação do território do DF, devido ao crescimento populacional e à ausência de oferta de moradia, principalmente para a classe média. Aumentaram as ocupações irregulares com invasões de terras públicas, em loteamentos clandestinos ofertados para a população desta classe, impossibilitada de arcar com os altos custos de moradia no Plano Piloto. Isto levou a população do DF a ser segregada espacialmente das áreas centrais, onde as populações de menor renda ficam cada vez mais afastadas das áreas centrais, onde desenvolvem a maior parte de suas atividades. Assim, assentamentos urbanos periféricos a Brasília foram desenvolvidos, denominados popularmente de cidade satélites (ou cidades dormitório) e posteriormente passaram a formar parte das Regiões Administrativas (RA). 
Os dados da Pesquisa Domiciliar O/D 2000 e informações das características do espaço urbano do Distrito Federal no ano 2000, obtidas por intermédio de um Sistema de Informações Geográficas (SIG), são utilizados no presente estudo de caso.

\subsection{Definição dos vetores de expansão urbana}

Para definição dos vetores de expansão urbana foram inseridas as zonas de tráfego georeferenciadas no ambiente SIG onde os eixos foram identificados. Aplicando uma análise de geoprocessamento da imagem satélite do Distrito Federal no ano 2000 e dados de Anjos (2010), foram identificados os seguintes eixos de expansão urbana: i) Vetor 1 - eixo Sobradinho; ii) Vetor 2 - eixo Planaltina; iii) Vetor 3 - eixo Taguatinga - Ceilandia; iv) Vetor 4 - eixo Taguatinga - Samambaia; e, v) Vetor 5 - eixo Gama. A sobreposição de layers facilitou a seleção de zonas de tráfego para formar os vetores de expansão urbana utilizados para a análise. Como critérios de seleção de zonas de tráfego e formação dos vetores de expansão urbana, foram considerados os seguintes:

- Primeiro, foram selecionadas unicamente as zonas de tráfego dentro do alcance dos eixos identificados.

- Segundo, foram selecionadas unicamente as zonas de tráfego com densidade residencial dentro de seus atributos. As zonas de tráfego com densidade residencial igual a zero foram descartadas.

Assim, a Figura 3 apresenta os vetores de expansão urbana formados. 


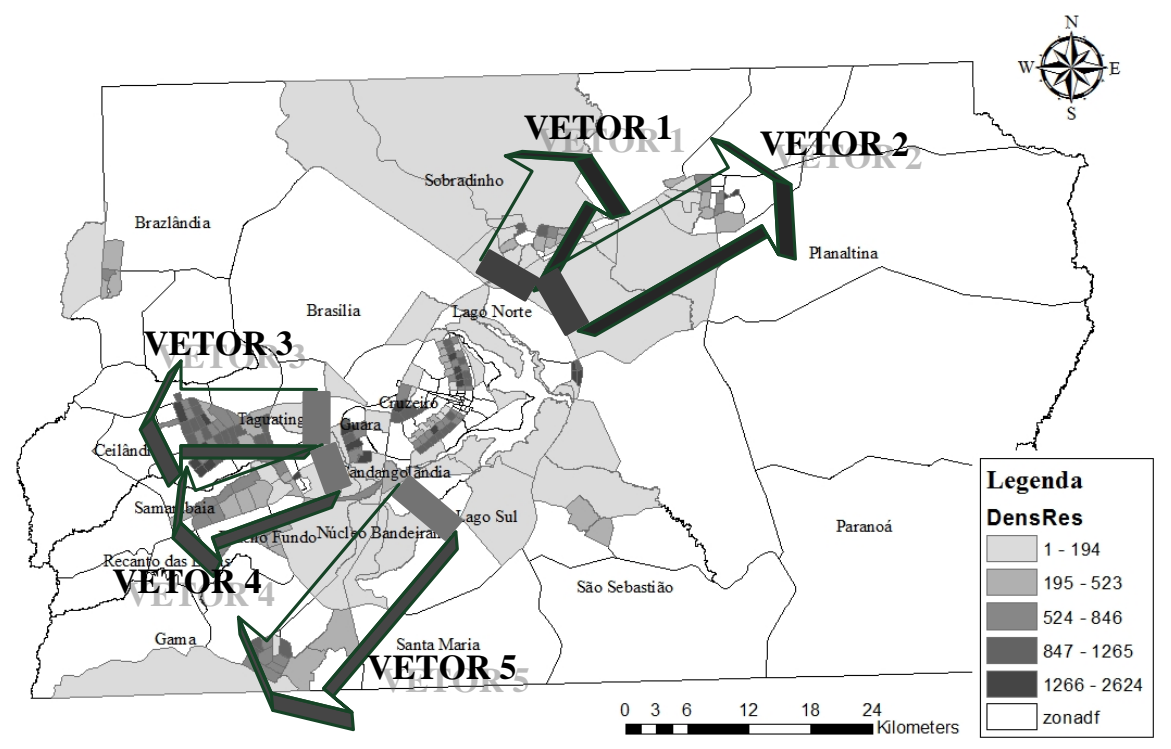

Figura 3 - Vetores de expansão

\subsection{Variáveis indicadoras}

A base de dados, contendo as zonas de tráfego, foi classificada por vetor de expansão urbana, sendo ao todos cinco: o primeiro constituído por 18 zonas de tráfego que produzem em total 362.645 viagens; o segundo, com 15 zonas de tráfego que produzem 286.386 viagens; o terceiro, com 42 zonas de tráfego que produzem 2.197.066 viagens; o quarto, constituído por 19 zonas de tráfego que produzem 871.705 viagens; e o quinto, com 17 zonas de tráfego que produzem 842.044 viagens. A partir desses vetores de expansão urbana, foram construídas as variáveis indicadoras para os construtos latentes.

Fatores espaciais da expansão urbana como variáveis indicadoras foram utilizadas: a densidade residencial $\left(D E N_{R}\right)$, e a densidade de uso de solo misto $\left(M I X_{U S}\right)$. Na Figura 4, observa-se a distribuição espacial da densidade residencial por zona de tráfego no DF, dividida em cinco classes. Além do Plano Piloto, existe uma concentração de zonas de alta densidade no eixo Taguatinga - Ceilândia, cujo eixo forma o vetor de expansão três. Os vetores 1 e 2 apresentam as mais baixas densidades.

No caso do indicador de densidade de uso de solo misto $\left(M I X_{U S}\right)$ a Figura 5 apresenta a distribuição espacial desta variável nas zonas de tráfego selecionadas para os vetores de expansão, divididos em quatro classes: i) valores de 0,091 até 0,239 ; ii) 0,240 até 0,382 ; iii) 0,383 até 0,602 ; e, iv) 0,603 até 0,930 . 


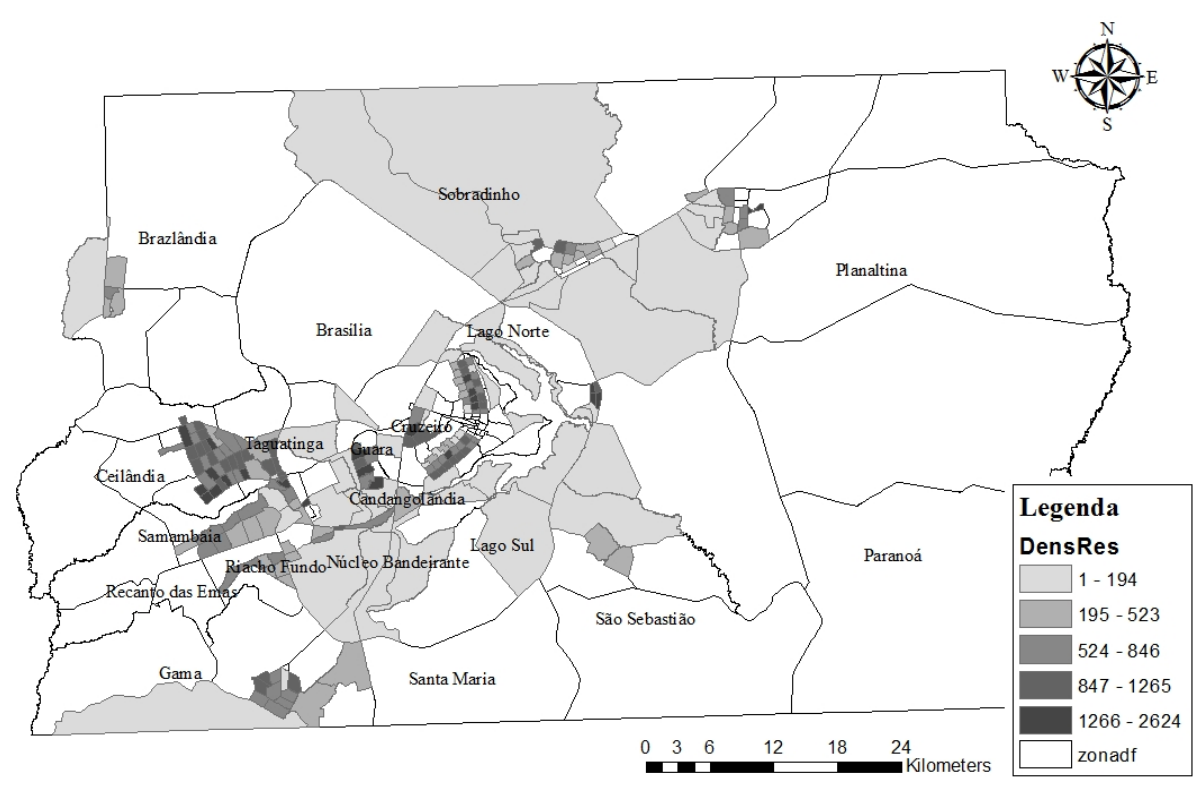

Figura 4 - Mapa temático da densidade residencial no Distrito Federal ano 2000

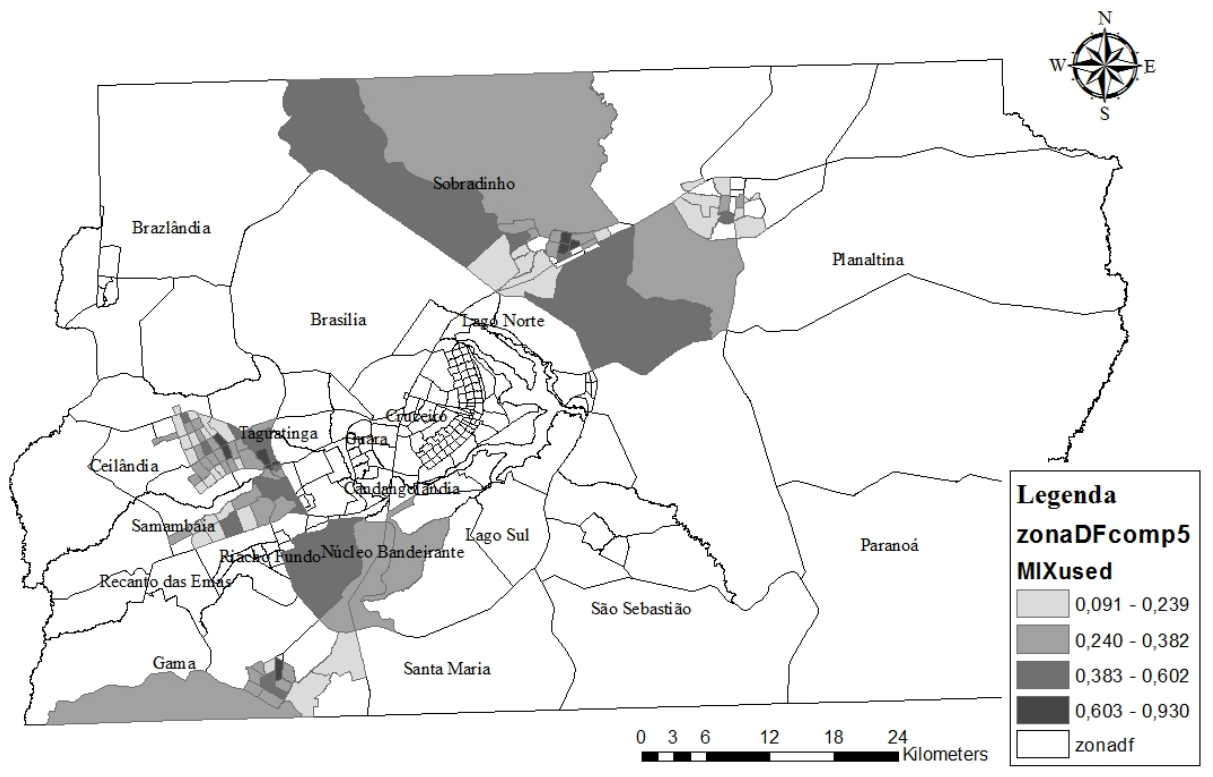

Figura 5 - Mapa temático do $M I X_{U S}$ nas zonas de tráfego selecionadas do DF ano 2000

Características socioeconômicas: Para o vetor de fatores socioeconômicos foram utilizadas as variáveis indicadoras: Número de famílias com dois carros ou mais e Número de famílias com renda acima de 20 salários mínimos. Os dados para essas variáveis foram agregadas por zona de tráfego. A Tabela 3 apresenta os parâmetros descritivos das variáveis indicadoras agrupadas por vetor de expansão urbana. 
Padrões de viagens baseados em atividades: Os padrões HW (residência - trabalho) e HS (residência - estudo) representam a maioria das viagens realizadas nos vetores, considerando como base a residência (Tabela 4). O padrão HW apresenta os maiores percentagens de viagens urbanas para os vetores 1,2 , e 3 . Mas, no caso dos vetores 4 e 5 , o padrão HS apresenta maiores percentagens.

Observa-se que o Plano Piloto é a região de maior atração de viagens HW, considerando a distribuição espacial das linhas de desejo de viagens dos cinco vetores na Figura 6. Taguatinga representa a segunda região com maior atração de viagens HW para os vetores 3 , 4, e 5, observando-se que existem também viagens internas entre os vetores de expansão urbana. Fenômenos similares de viagens ao Plano Piloto e viagens internas podem ser observadas para os vetores 1 e 2 .

Os padrões HM (residência - saúde) e HA (residência - outras atividades) apresentam percentagens menores, portanto existem poucos residentes nos vetores de expansão que realizam estas atividades como principais. Portanto, é possível que saúde e outras atividades sejam realizadas como atividades secundárias, complementares às atividades principais trabalho e estudo.

Tabela 3 - Parâmetros descritivos das variáveis indicadoras características socioeconômicas

\begin{tabular}{lccccc}
\hline Variável & No obs. & Media & Minimo & Máximo & Std.dev \\
\hline \hline Número de familias com renda acima de & 20 & salarios & mínimos & \\
\hline Vetor 1 & 18 & 1193,000 & 0 & 8130 & 1973,602 \\
Vetor 2 & 15 & 379,667 & 0 & 1384 & 475,025 \\
Vetor 3 & 42 & 1318,500 & 0 & 8569 & 1839,955 \\
Vetor 4 & 19 & 1327,053 & 0 & 8923 & 2227,023 \\
Vetor 5 & 17 & 1919,471 & 0 & 7453 & 1917,273 \\
\hline Número de familias com dois carros o mais & & & \\
\hline Vetor 1 & 18 & 2705,278 & 0 & 11174 & 2787,558 \\
Vetor 2 & 15 & 796,800 & 0 & 2568 & 811,649 \\
Vetor 3 & 42 & 3459,119 & 0 & 10456 & 2841,156 \\
Vetor 4 & 19 & 2151,316 & 0 & 7187 & 1794,949 \\
Vetor 5 & 17 & 3650,941 & 0 & 8876 & 2624,319 \\
\hline
\end{tabular}


Tabela 4 - Frequência de padrões de viagens baseadas em atividades

\begin{tabular}{ll|cc}
\hline $\begin{array}{c}\text { Padrões de } \\
\text { viagens B.A. }\end{array}$ & $\begin{array}{c}\text { Nro. } \\
\text { Viagens }\end{array}$ & $\begin{array}{c}\text { (\%) Relativo ao total de } \\
\text { viagens baseados na } \\
\text { residência (H) por vetor }\end{array}$ \\
\hline \multirow{2}{*}{ Vetor 1 } & HW & 204723 & 52 \\
& HS & 192084 & 48 \\
\hline \multirow{2}{*}{ Vetor 2 } & HW & 203031 & 51 \\
& HS & 198082 & 49 \\
\hline \multirow{2}{*}{ Vetor 3 } & HW & 1058349 & 51 \\
& HS & 1035170 & 49 \\
\hline \multirow{2}{*}{ Vetor 4 } & HW & 445092 & 45 \\
& HS & 543114 & 55 \\
\hline \multirow{2}{*}{ Vetor 5 } & HW & 453969 & 46 \\
& HS & 542894 & 54 \\
\hline
\end{tabular}

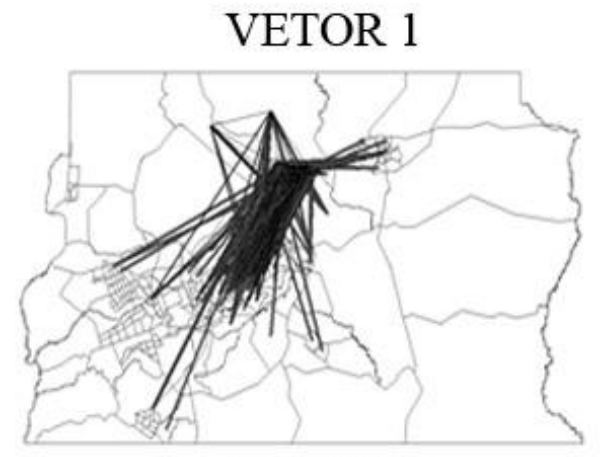

VETOR 3
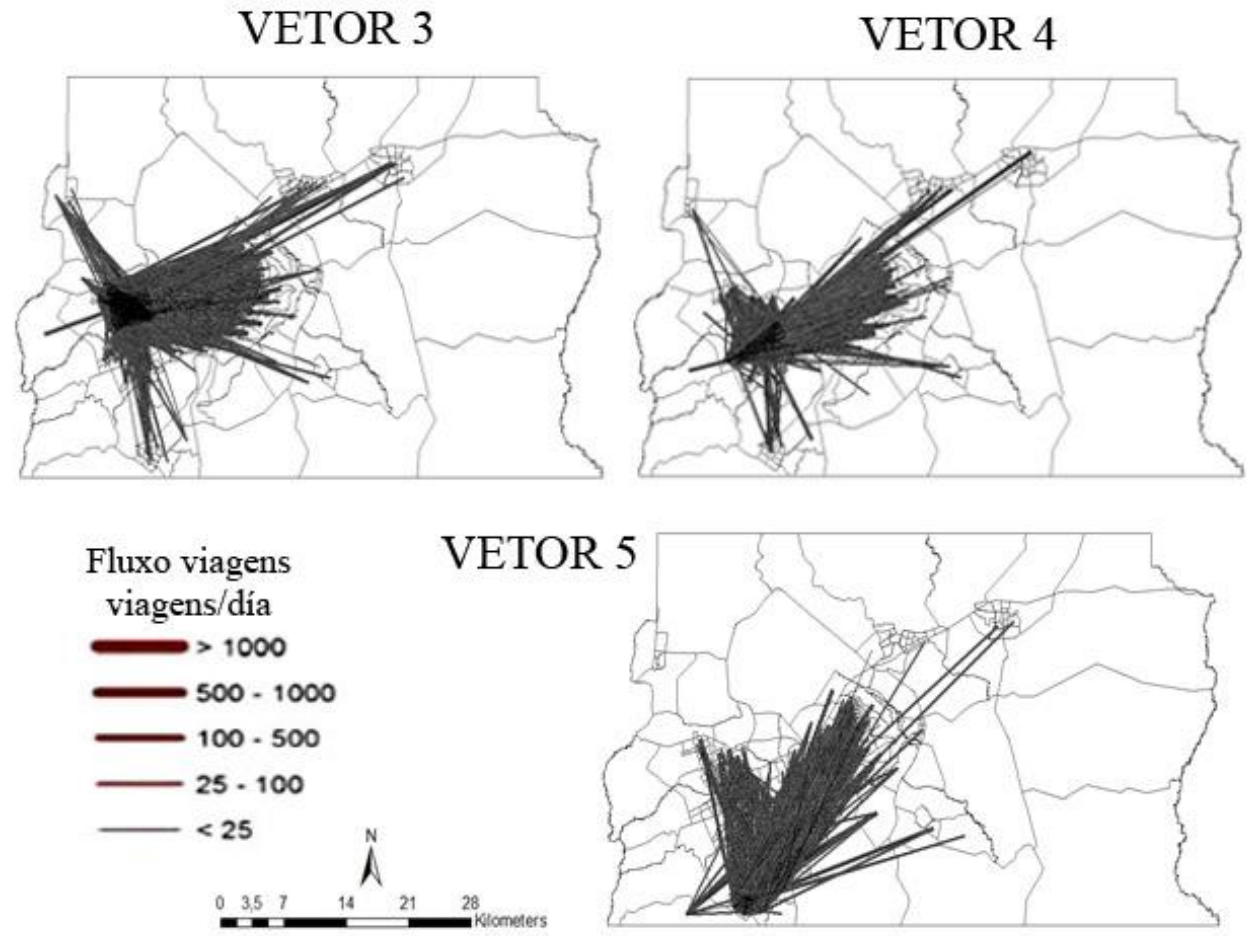

Figura 6 - Fluxos de viagens HW para cada vetor de expansão urbana 


\section{Análise dos resultados}

Nesta seção são apresentados os resultados da modelagem. A Figura 7 apresenta o modelo ABTUS com os valores dos parâmetros padronizados. A modelagem foi realizada considerando duas variáveis latentes exógenas cada uma com dois indicadores de mensuração, e uma variável latente endógena também com dois indicadores de mensuração. Os resultados da avaliação global do modelo são apresentados na Tabela 5.

O modelo foi estimado utilizando a Máxima Verossimilhança. Em resumo, os resultados obtidos nos índices de avaliação global do modelo proporcionam suficiente evidência para aceitar a boa qualidade de ajuste do modelo ABTUS.

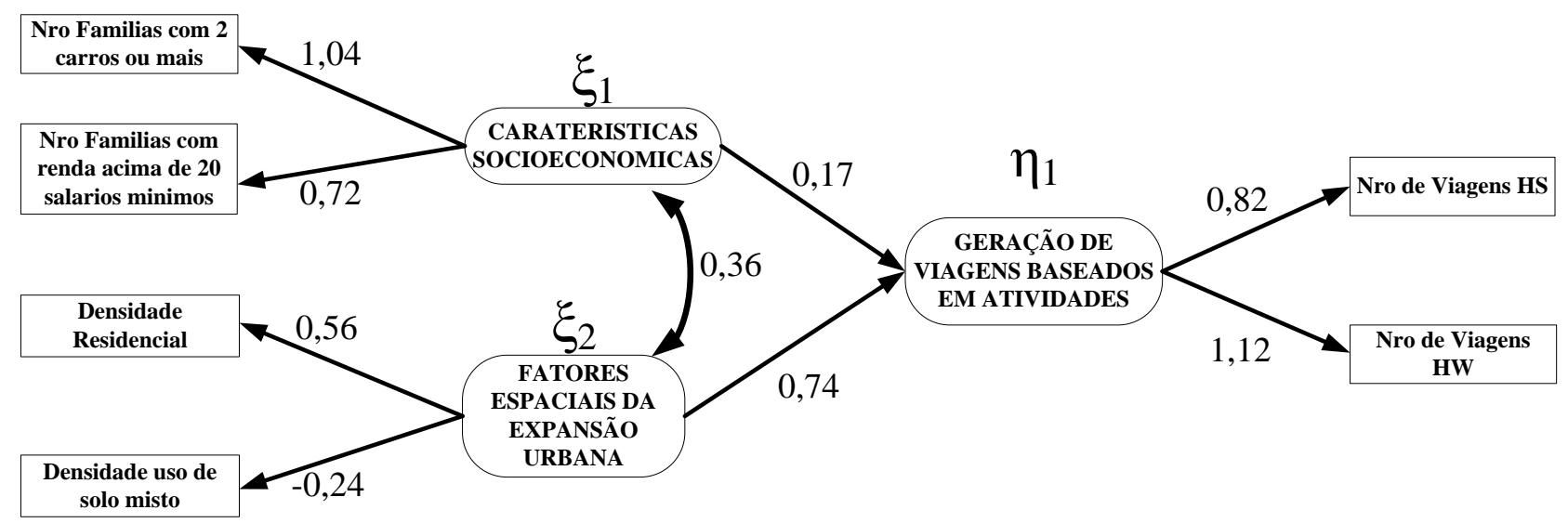

Figura 7 - ABTUS com parâmetros padronizados

$\mathrm{Na}$ avaliação local do modelo foram analisadas todas as relações entre as variáveis com o propósito de analisar a relação entre a expansão urbana e os padrões de viagens baseadas em atividades. Na Tabela 6 são apresentados os parâmetros padronizados, a fim de medir a importância de explicação das variáveis. A Figura 7 também apresenta as cargas fatoriais padronizadas para cada caminho de relação entre indicadores e variáveis latentes exógenas, e entre variáveis exógenas e a variável endógena. 


\section{Tabela 5 - Índices de avaliação global do modelo}

\begin{tabular}{lc}
\hline & $\begin{array}{c}\text { Bondade de Ajuste do } \\
\text { modelo }\end{array}$ \\
$\begin{array}{ll}\text { Tamanho da amostra } \\
\text { Valor Qui-quadrado }\end{array}$ & 111 \\
& 9,976 \\
Graus de liberdade & $(\mathrm{P}=0,190)$ \\
Índice de Bondade de Ajuste (GFI) & 7 \\
Índice Ajustado de Bondade de Ajuste & 0,973 \\
(AGFI) & 0,919 \\
Índice de Ajuste Normalizado (NFI) & 0,974 \\
Índice de Ajuste Comparativo (CFI) & 0,992 \\
RMSA & 0,062 \\
& $(\mathrm{LO} 90 \%=0,000 ;$ \\
& $\mathrm{HI} 90 \%=0,142)$ \\
\hline
\end{tabular}

Tabela 6 - Parâmetros estimados de efeitos totais padronizados

\begin{tabular}{lcc}
\hline Trom & To & \\
& $\begin{array}{c}\text { Fatores espaciais da } \\
\text { expansão urbana }\end{array}$ & $\begin{array}{c}\text { Características } \\
\text { socioeconômicas de viagens } \\
\text { por atividade }\end{array}$
\end{tabular}

\section{Latentes exógenas}

Fatores espaciais da expansão urbana da Características socioeconômicas

$\begin{array}{lll}\gamma_{12} & 0,741 & * * * \\ \gamma_{11} & 0,167 & * * *\end{array}$

\section{Exógenas}

Densidade residencial

Densidade uso de solo

$\begin{array}{ll}0,556 & * * * \\ -0,242 & * *\end{array}$

Número de familias com renda acima de 20 salarios mínimos

Número de familias com dois carros o mais

Padrão de viagens HW 


\subsection{Fatores espaciais da expansão urbana}

O impacto dos Fatores espaciais da expansão urbana, parâmetro $\gamma_{12}$, na geração de viagens por atividade é de 0,741 , satisfazendo o teste de hipótese a um nível de confiança de $1 \%$. O que significa que os fatores espaciais da expansão urbana influenciam positivamente a geração de viagens por atividade na cidade de Brasília.

O efeito total da variável indicadora de densidade residencial, $D E N_{R}$, foi positivo o que indica que os Fatores espaciais da expansão urbana de Brasília influenciam na existência de um maior adensamento residencial nas expansões urbanas. A configuração e políticas urbanas da cidade direciona aos habitantes a procurar moradia nas expansões urbanas.

Para caso da variável indicadora de densidade de uso de solo misto, $M I X_{U S}$, apresenta uma relação negativa. Os fatores espaciais da expansão urbana diminuem a intensidade do tipo de uso de solo, isto é as expansões urbanas de Brasília tendem a ser mais de uso residencial que outros tipos de usos de solo, o que produz viagens em procura de atividades não encontradas na expansão urbana.

\subsection{Características socioeconômicas}

O impacto das características socioeconômicas sobre a geração de viagens por atividade $\left(\gamma_{11}\right)$ apresenta um valor de 0,167 , satisfazendo o teste de hipótese a um nível de confiança de $1 \%$. O que significa que as características socioeconômicas influenciam positivamente na geração de viagens por atividade em 0,167 na cidade de Brasília.

Os efeitos totais das variáveis indicadoras são significativos a um nível de confiança de $1 \%$. O número de famílias que possuem dois carros ou mais é influenciado positivamente pela variável latente de características econômicas de Brasília na expansão urbana. Assim, o processo socioeconômico do setor público e a restrita moradia da cidade produziram que as famílias procurem moradia nas expansões urbanas e utilizem o carro como o principal médio de deslocamento.

No caso do número de famílias com renda superior a 20 salários mínimos, o valor estimado foi positivo. O que indica que a configuração socioeconômica da expansão urbana de Brasília 
apresenta uma tendência de famílias com uma elevada renda que residem nestas expansões urbanas.

A expansão urbana de Brasília apresenta características de adensamento residencial e maior motorização individual das famílias. Os resultados obtidos claramente refletem que isso é devido a influencia de processos de produção de espaço e socioeconômicos característicos da cidade. Os quais foram introduzidos na modelagem como variáveis latentes. Além disso, os dois processos influenciam positivamente na geração de viagens por atividade. Essa influencia é dada por uma relação de interdependência entre os efeitos das características espaciais da expansão urbana e as características socioeconômicas dos indivíduos que moram na expansão urbana. Essa relação pode ser interpretada pelo parâmetro $\phi$ da covariância.

Assim, os resultados encontrados permitem entender a importância da relação estrutural da covariância entre as características espaciais urbanas e as características dos indivíduos na geração de viagens por atividade. Para o caso de Brasília essa relação apresenta uma interdependência positiva entre fatores espaciais da expansão urbana e características socioeconômicas dos indivíduos residentes nas expansões urbanas que influenciam na geração de viagens por trabalho e estudo. Essa covariância representa a historia completa da evolução urbana da cidade de Brasília.

\section{Conclusão}

Este trabalho teve como objetivo analisar a relação dos efeitos espaciais entre expansão urbana e padrões de viagens baseadas em atividades, mediante a utilização da modelagem com equações estruturais. Foi elaborado um modelo causal "ABTUS (Activity-Based Travels of Urban Sprawl)" que não relaciona os fatores da expansão urbana de forma direta com os padrões de viagens baseados em atividades, mas estabelece uma relação indireta através da formação de construtos latentes. O modelo foi testado em caso de estudo de Brasilia-Distrito Federal para o ano 2000. A partir dos resultados obtidos cinco vetores de expansão urbana foram identificados e os padrões de viagens mais representativos foram baseados nas sequências de atividades Residência Trabalho e Residência Estudo. 
Como observado, o processo de expansão urbana e a distribuição das atividades no espaço urbano afetam o sistema de transporte. Assim, o modelo ABTUS traz o entendimento por meio da relação entre o processo de expansão urbana e a distribuição das atividades no espaço urbano, e como isso afeta a geração de viagens, gerando impactos diretos no sistema de transporte. O modelo ABTUS pode auxiliar o planejamento, provendo um bom entendimento da relação entre a expansão urbana e os padrões de viagens baseadas em atividades. Além disso, acredita-se que esse modelo possibilite prever a resposta dos indivíduos perante mudanças no sistema de transporte, ou nos subsistemas de atividades, bem como no ambiente socioeconômico e demográfico.

Para o caso de Brasília a expansão urbana esta fortemente marcada pelo adensamento residencial e características socioeconômicas de famílias com dois o mais carros. Assim, a centralização dos empregos e o restito espaço de moradia no centro da cidade, produzem maiores viagens nas expansões urbanas por trabalho e estudo. Claramente o efeito da densidade residencial e centralização dos empregos influenciam na geração de viagens nas expansões urbanas como o caso de Estados Unidos e Europa, mas Brasília apresenta famílias que dependem do carro particular assim como famílias que utilizam transporte público e adensamentos residenciais mais concentrados.

A maioria das análises empíricas sobre expansão urbana e transporte considera uma abordagem baseada em uma relação linear entre a viagem, os fatores socioeconômicos e a forma urbana. As pesquisas têm se concentrado mais na correlação estatística entre fatores relevantes do que em relações causais. Isso leva a mais uma vez afirmar a necessidade do entendimento do comportamento de viagem como resultante do processo de expansão urbana, o qual está sujeito a uma ralação de covariância estrutural entre fatores de espaço urbano, socioeconômicos. A partir desse processo, é possível identificar fatores que mais se ajustem a realidade brasileira.

Um condicionante negativo foi a mensuração da expansão urbana para elaboração das variáveis indicadoras. Existem muitos trabalhos sobre expansão urbana, mas poucos têm enfoque em suas causas ou fornecem indicadores para a sua mensuração. Todavia, há técnicas mais precisas e automatizadas para esse tipo de levantamento, porém essas exigem algum conhecimento especializado. Considerando a dimensão da análise da área de estudo, seria necessário um tempo maior para a aplicação dos indicadores. 
Assim, ABTUS é um modelo que pode ser melhorado aplicando mais indicadores de mensuração da expansão urbana. Como também a inclusão de variáveis de acessibilidade espacial temporal. Contudo, o método desenvolvido para aplicação do ABTUS no caso de estudo permite também a sua aplicação em outros estudos de caso, bem como a formulação de um novo modelo, de forma atender os mesmos fins estabelecidos neste trabalho.

Como conclusão final pode-se afirmar que os resultados apresentados neste modelo são evidências em favor do uso regulado do solo e intervenções normativas nas expansões urbanas como ações para mudar o comportamento de viagens. $\mathrm{O}$ aprofundamento dessa relação pode oferecer subsídios para auxiliar na abstração de padrões e análise de demanda de viagens; ou seja, ordem e tendências que podem nortear, inclusive, processos de tomada de decisão, implantação de novos projetos, solução de problemas de congestionamentos e mobilidade, e desafios propostos para a sustentabilidade do transporte.

\section{Referências}

Anjos, R. S. (2010) Monitoramento do crescimento e vetores de expansão urbana de Brasília. Brasília 50 anos de capital a metrópole. UnB, Brasília, 490p.

Camagni, R. ; Gibelli, M.C. ; Rigamonti, P. (2002). Urban mobility and urban form: the social and environmental costs of different patterns of urban expansion. Ecological Economics, vol 40, 199-216.

Castells, M. (1974). La cuestion Urbana. Madrid: Siglo veintiuno editores S.A.

Cervero, R. (1998) The Transit Metropolis: a global inquiry. Washington: Island Press.

Cervero, R., Kockelman, K. (1997). Travel demand and the 3D's: density, diversity, and design. Transportation Research D, vol. 2, n. 3, pp. 199-219.

Duany, A., Plater-Zyberk, E., Speck, J. (2000). Suburban nation: the rise of sprawl and the decline of the American dream. New York: North Point Press.

Ewing, R. (1997). Is Los Angeles-style sprawl desirable? Journal of the American Planning Association, vol. 63, n. 1, pp. 107-126.

Frank, L. D., Pivo, G. (1994). Impacts of mixed use and density on utilization of three modes of travel: single occupant vehicle, transit, and walking. Transportation Research Record, n. 1466, pp. 44 52.

Friedman, B., Gordon, S.P., Peers, J.B. (1994). Effect of neo-traditional neighborhood design on travel characteristics. Transportation Research Record, n. 1466, pp. 63-70.

García-Palomares J.C. (2010). Urban sprawl and travel to work: the case of the metropolitan area of Madrid. Journal of Transport Geography, n.18, pp. 197-213.

Golob, T.F. (2008). "Structural equation modeling for travel behavior research". Transportation Research, B - Methodological, n. 37, pp. 1-25. 
Giuliano, G., Narayan, D. (2002). Another look at travel patterns and urban form: the US and Great Britain. Urban Studies, vol. 40, n. 11, pp. 2295-2312.

Kenworthy, J. (1995). Automobile dependence in Bangkok: an international comparison with implications for planning policies. World Transport Policy and Practice, vol. 3, n. 1, pp. 31-41.

Gakenheimer, R. (1999) Urban mobility in the developing world. TransportationResearch A, vol. 33, n. 7, pp. 671-689.

Newman, P. W. G., \& Kenworthy, J. R. (1989) Gasoline consumption and cities: a comparison of U.S. cities with a global survey. Journey of American Planners Association, vol. 55, n. 1, pp. 24-37.

Schwanen, T., Dieleman, F.M., Dijst, M. (2001) Travel behaviour in Dutch monocentric and polycentric urban systems. Journal of Transport Geography, vol. 9, n. 3, pp. 173-185.

Travisi Chiara M., Camagni Roberto, Nijkamp Peter (2010) Impacts of urban sprawl and commuting: a modelling study for Italy. Journal of Transport Geography, vol.18, pp. 382-392.

Zhao Pengjun. (2010). Sustainable urban expansion and transportation in a growing megacity: Consequences of urban sprawl for mobility on the urban fringe of Beijing. Habitat International, vol. 34, pp. 236-243. 